\title{
Avaliação da qualidade da dieta de pacientes diabéticos tipo 2 atendidos no Ambulatório de Nutrição da Universidade Federal de Pelotas
}

\author{
Evaluation of the quality of the diet of type 2 diabetic \\ patients attended at the Nutrition Outpatient Clinic of the \\ Federal University of Pelotas
}

Larissa Barz de Vargas', Olivia Farias Santos², Larissa Sander Magalhães ${ }^{3}$, Débora Simone Kilpp ${ }^{4}$, Renata Abib Bertacco ${ }^{5}$, Anne Y Castro Marques ${ }^{6}$, Lúcia Rota Borges ${ }^{7}$

${ }^{7}$ Graduanda do curso de Nutrição pela Universidade Federal de Pelotas

${ }^{2}$ Nutricionista pela Universidade Federal de Pelotas

${ }^{3}$ Graduanda do curso de Nutrição da Universidade Federal de Pelotas

${ }^{4}$ Nutricionista do Serviço de Atenção Domiciliar (PIDI/Melhor em Casa) do Hospital-Escola da Universidade Federal de Pelotas (UFPEL); no Ambulatório do Centro de Pesquisas em Saúde Dr. Amílcar Gigante e no Serviço de Nutrição do Hospital-Escola da UFPEL/EBSERH ${ }^{5}$ Nutricionista, Especialização em Nutrição com ênfase em aterosclerose, mestre e doutora em Ciências Biológicas: Bioquímica, Pós-doutorado na área de Medicina. Professora Adjunta da Faculdade de Nutrição da Universidade Federal de Pelotas

${ }^{6}$ Doutora em Alimentos e Nutrição / UNICAMP. Professora adjunta da Faculdade de Nutrição / UFPEL

${ }^{7}$ Nutricionista, Professor Adjunto do Departamento de Nutrição da Faculdade de Nutrição da Universidade Federal de Pelotas. Doutora em Ciência e Tecnologia de Alimentos pela Universidade Federal de Pelotas. Mestre em Saúde e Comportamento pela Universidade Católica de Pelotas.

E-mail para contato: Larissa Barz de Vargas - laribdevargas@gmail.com

\section{Resumo}

Objetivo: Avaliar a qualidade da dieta de pacientes diabéticos tipo 2 atendidos no Ambulatório de Nutrição da Universidade Federal de Pelotas. Método: Estudo transversal com pacientes diabéticos tipo 2, maiores de 18 anos, atendidos no ambulatório de nutrição da Universidade Federal de Pelotas. Foram avaliados de forma retrospectiva, dados obtidos a partir dos prontuários dos pacientes, sendo traçado o perfil clínico, demográfico e nutricional. Para avaliar a qualidade da dieta foi aplicado o Índice de Qualidade da Dieta Revisado, a partir do recordatório de 24 horas dos pacientes na primeira consulta. Resultados: Foram avaliados 221 diabéticos tipo 2, sendo a maioria do sexo feminino $(67,42 \%)$ e obeso $(59,55 \%)$. A média geral do Índice de Qualidade da Dieta Revisado foi de 60,63 $\pm 9,88$ pontos. Os componentes com menor contribuição para a média do escore total foram frutas integrais, vegetais totais e leguminosas, cereais integrais, sódio, frutas totais e leite e derivados. Indivíduos idosos obtiveram escores significativamente maiores, além disso, houve correlação negativa entre o índice de qualidade da dieta e o índice de massa corporal, evidenciando que, quanto maior o índice de massa corporal menor o escore e pior a qualidade da dieta. Conclusão: Verificou-se inadequação na qualidade da dieta de pacientes diabéticos, principalmente entre aqueles que apresentaram sobrepeso e/ou obesidade, ressaltando a necessidade de intervenções nutricionais adequadas para essa população, visando à melhora de 
hábitos alimentares, com repercussões positivas no controle glicêmico e na qualidade de vida dos mesmos.

Palavras-chave: Diabetes Mellitus. Consumo de alimentos. Inquéritos alimentares. Dieta.

\begin{abstract}
Objective: To evaluate the quality of the diet of type 2 diabetic patients attended at the Nutrition Outpatient Clinic of the Federal University of Pelotas. Method: Cross-sectional study with type 2 diabetic patients, over 18 years of age, attended at the nutrition clinic of the Federal University of Pelotas. Retrospective data were obtained from the patients' charts, and the clinical, demographic and nutritional profiles were traced. To evaluate the quality of the diet, the Revised Diet Quality Index was applied, based on the patients' 24-hour recall at the first visit. Results: A total of 221 type 2 diabetic patients were evaluated, the majority being female (67.42\%) and obese (59.55\%). The overall mean of the Revised Diet Quality Score was $60.63 \pm 9.88$ points. The components with the lowest contribution to the mean of the total score were whole fruits, total vegetables and legumes, whole grains, sodium, total fruits and milk and dairy products. Elderly subjects had significantly higher scores; in addition, there was a negative correlation between diet quality index and body mass index, evidencing that the higher the body mass index the lower the score and the worse the quality of the diet. Conclusion: There was an inadequacy in the quality of the diet of diabetic patients, especially among patients who were overweight and / or obese, emphasizing the need for adequate nutritional interventions for this population, aiming at improving eating habits, with positive repercussions on glycemic control and their quality of life.
\end{abstract}

Keywords: Diabetes Mellitus. Food consumption. Food Inquiries. Diet.

\title{
INTRODUÇÃO
}

O Diabetes Mellitus (DM) é considerado um dos maiores e crescentes problemas de saúde pública do mundo, estando entre as dez principais causas de óbitos, sobretudo em países em desenvolvimento. Estima-se que, em 2030, o DM será a sétima causa de óbito, entre as doenças crônicas não transmissíveis (DCNT) e segundo dados da International Diabetes Federation (IDF), o Brasil é o quinto país do mundo com o maior número de diabéticos, apresentando 16,8 milhões de indivíduos com a doença $\mathrm{a}^{1-4}$.

Trata-se de uma síndrome metabólica complexa, de etiologia múltipla, caracterizada por hiperglicemia persistente, resultante de defeitos na ação e/ou secreção de insulina. Dentre os tipos existentes, o diabetes mellitus tipo 2 (DM2) corresponde por aproximadamente, 90 a $95 \%$ de todos os casos da doença ${ }^{5}$.

O DM apresenta alta morbimortalidade, com perda importante na qualidade de vida, podendo ocasionar maior incidência de doenças cardio e cerebrovasculares, 
cegueira, insuficiência renal e amputações não traumáticas de membros inferiores ${ }^{6}$. Assim, é previsto maior número de hospitalizações e maior utilização dos serviços de saúde representando uma sobrecarga nos próximos anos para os sistemas de saúde de todos os países7 ${ }^{7}$ Além disso, o DM causa um problema social importante, em decorrência da perda de produtividade no trabalho, da aposentadoria precoce e da mortalidade prematura ${ }^{6}$.

Reduzir o impacto do DM2 significa reduzir a incidência da doença, controlando os fatores envolvidos na sua ocorrência, como por exemplo, a alimentação inadequada, o sedentarismo e o aumento da obesidade ${ }^{6,8,9}$. A literatura científica tem evidenciado a relação importante entre hábitos alimentares inadequados e a ocorrência de DM2, devido ao baixo consumo de alimentos ricos em fibras e ao elevado consumo de açúcares, carboidratos refinados, sódio e gorduras totais e saturadas ${ }^{10-12}$.

Em virtude do padrão alimentar ser um dos principais fatores etiológicos de DM2 e para o surgimento de outras DCNT, instrumentos como os índices dietéticos têm sido utilizados com a finalidade de avaliar a qualidade da dieta da população, para assim, conhecer o perfil alimentar dos indivíduos, possibilitando a realização de estratégias de intervenção e promoção à saúde, e ações de mudança de hábitos alimentares, acarretando na redução da incidência de comorbidades ${ }^{13}$.

Em 1995 foi desenvolvido por Kennedy et al. ${ }^{14}$, o Healthy Eating Index (HEI), baseado em recomendações dietéticas para a população norte-americana. Esse índice disponibiliza uma soma de componentes alimentares que permite a avaliação global da dieta ${ }^{14}$. O índice foi revisado periodicamente para incorporar os avanços de pesquisas do campo da Nutrição, tendo sua mais recente atualização no ano de 2010, quando passou a ter 12 componentes que avaliam o grau de atendimento às recomendações de consumo de alimentos e nutrientes ${ }^{15}$.

O HEI foi adaptado e validado para população brasileira por Fisberg et al. em $2004^{16}$, seguindo as recomendações da Pirâmide Alimentar proposta por Philippi et al. ${ }^{17}$, originando o Índice de Qualidade da Dieta (IQD). Entretanto com a publicação do Guia Alimentar para a População Brasileira em 2006, houve a necessidade de revisar o IQD, gerando o Índice da Qualidade da dieta revisado (IQD-R) proposto por Previdelli et al. ${ }^{18}$. O IQD-R permite analisar simultaneamente diferentes componentes da dieta, com base na densidade energética, avaliando sua qualidade, independentemente da quantidade de alimento consumido ${ }^{18}$. 
Considerando a alta incidência de DM2 na população e sua relação com hábitos alimentares inadequados, é de suma importância a avaliação da qualidade da dieta por meio do IQD-R, visto que o padrão alimentar desta população específica é interligado com o desfecho da doença, que acarreta consequências negativas para o indivíduo diabético e para a sociedade. Diante do exposto, este estudo teve como objetivo avaliar a qualidade da dieta de pacientes diabéticos tipo 2 atendidos no Ambulatório de Nutrição, da Universidade Federal de Pelotas.

\section{MÉTODO}

Trata-se de um estudo transversal, onde foram avaliados, de forma retrospectiva, dados da primeira consulta, obtidos dos prontuários dos pacientes portadores de DM2, atendidos no ambulatório de Nutrição do Centro de Referência em Diabetes e Hipertensão Arterial, no período de março de 2016 a março de 2018. Foram incluídos os dados de todos os pacientes maiores de 18 anos, de ambos os sexos, portadores de DM2, que possuíam todos os dados necessários para a realização do estudo.

Para traçar o perfil dos diabéticos atendidos no ambulatório, foram coletadas dos prontuários as seguintes variáveis: idade (em anos completos), sexo, procedência, escolaridade (ensino fundamental incompleto, ensino fundamental completo, ensino médio incompleto, ensino médio completo e superior completo), cor (branca e não branca), estado civil (com companheiro e sem companheiro), presença de outras comorbidades, peso, altura e índice de massa corporal (peso (kg) / altura $\left.\left(\mathrm{m}^{2}\right)\right)(\mathrm{IMC})$. Para a classificação do IMC em adultos foram utilizados os parâmetros estabelecidos pela World Health Organization (WHO) ${ }^{19}$ : IMC menor do que $18,5 \mathrm{~kg} / \mathrm{m}^{2}$ - baixo peso; IMC entre $18,5 \mathrm{~kg} / \mathrm{m}^{2}$ e $24,9 \mathrm{~kg} / \mathrm{m}^{2}$ - eutrofia; IMC entre $25 \mathrm{~kg} / \mathrm{m}^{2}$ e $29,9 \mathrm{~kg} / \mathrm{m}^{2}$ - sobrepeso; e IMC igual ou maior a $30 \mathrm{~kg} / \mathrm{m}^{2}$ - obesidade. E já para a classificação do IMC em idosos (>60 anos) foram utilizados os pontos de corte da Organização Pan-americana da Saúde (OPAS) ${ }^{20}$ : IMC menor do que $23 \mathrm{~kg} / \mathrm{m}^{2}$ baixo-peso; IMC entre $23 \mathrm{~kg} / \mathrm{m}^{2}$ e $28 \mathrm{~kg} / \mathrm{m}^{2}$ - eutrofia; IMC entre $28 \mathrm{~kg} / \mathrm{m}^{2}$ e $30 \mathrm{~kg} / \mathrm{m}^{2}$ - sobrepeso e IMC maior que $30 \mathrm{~kg} / \mathrm{m}^{2}$ - obesidade.

O consumo alimentar foi avaliado por meio do recordatório de 24 horas, obtido na primeira consulta dos pacientes. As informações coletadas por meio dos recordatórios foram analisadas com auxílio do software Nutriquanti ${ }^{\circledR}$ e a qualidade da dieta foi quantificada a partir da análise da pontuação do IQD-R para a população brasileira, baseado no Guia Alimentar ${ }^{18,21}$. 
O instrumento avalia a dieta de acordo com doze componentes, dos quais nove são grupos alimentares (frutas totais; frutas integrais; vegetais totais e leguminosas; vegetais verdes-escuros, alaranjados e leguminosas; cereais totais; cereais integrais; leite e derivados; carnes, ovos e leguminosas; óleos), dois são nutrientes (gordura saturada; sódio) e o último representa a soma do valor energético proveniente da ingestão de gordura sólida, álcool e açúcar de adição (componente Gord_AA $)^{18}$.

Os componentes de um a nove avaliam a adequação, já os componentes de dez a doze a moderação do consumo alimentar; esta moderação é avaliada em sentido oposto, isto é, o aumento do consumo reflete negativamente, enquanto o consumo de alimentos de um a nove, quanto maior o consumo vai refletir positivamente no resultado final. A pontuação mínima é atribuída ao consumo zero (componentes um a nove) ou ao consumo acima do limite preconizado (componentes dez a doze), enquanto a pontuação máxima é estabelecida atingindo ou ultrapassando o valor recomendado de ingestão do consumo (componente de um a nove) ou consumo dentro do limite preconizado (componente de dez a doze). O resultado é representado por uma pontuação que varia de 0 a 100 pontos, logo, pontuações mais altas, representam melhor qualidade da dieta ${ }^{18}$. Com o intuito de classificar essa qualidade da dieta com mais clareza foi utilizado à classificação do IQD anterior ${ }^{16}$, que classifica a dieta em três categorias: abaixo ou igual a 40 pontos - dieta "inadequada"; entre 41 e 64 pontos - dieta que "necessita de modificação"; e igual ou superior a 65 pontos - dieta "saudável".

Os dados foram inseridos em um banco de dados no programa Excel ${ }^{\circledR}$ versão 2007 para Windows e posteriormente exportados para o programa Stata ${ }^{\circledR}$ versão 12.0, sendo realizada a análise das variáveis estudadas, com apresentação de médias e desvios-padrão ou proporções, conforme a natureza da variável. As comparações e testes de associação foram realizados pelos testes de qui-quadrado ou Exato de Fischer. Para verificar as diferenças de médias foi utilizado o teste t-Student. A associação entre qualidade da dieta e índice de massa corporal foi averiguada através da correlação de Pearson. O nível de significância para todas as análises foi de $5 \%$ $(\mathrm{p}<0,05)$.

Este estudo é um sub estudo transversal de um projeto previamente aprovado pelo Comitê de Ética em Pesquisa da Faculdade de Medicina da Universidade Federal de 
Pelotas (UFPel), sob o número 1.659.342. O trabalho seguiu as determinações da resolução $n^{0} 466 / 2012$ para pesquisa com seres humanos.

\section{RESULTADOS}

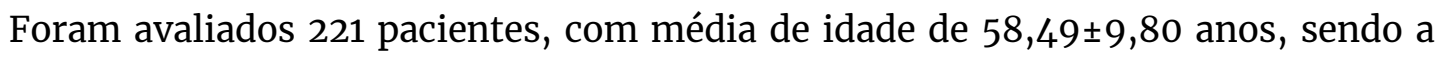
amostra composta por maior proporção de mulheres $(67,42 \%)$, procedentes de Pelotas (90,50\%), adulta (53,39\%), possuíam companheiro $(63,80 \%)$, eram da cor branca $(81,45 \%)$, apresentavam ensino fundamental completo ou médio incompleto $(79,19 \%)$ e tinham diagnóstico de hipertensão arterial associada ao diabetes $(82,81 \%)$ (Tabela 1$)$.

Tabela 1 - Descrição da amostra segundo o perfil sociodemográfico dos pacientes diabéticos tipo 2 atendidos no Centro de Diabetes e Hipertensão da UFPel em Pelotas-RS, 2019 ( $n=221)$

\begin{tabular}{|c|c|c|}
\hline Variável * & $\mathbf{N}$ & $\%$ \\
\hline $\begin{array}{l}\text { Sexo } \\
\text { Masculino } \\
\text { Feminino }\end{array}$ & $\begin{array}{c}72 \\
149\end{array}$ & $\begin{array}{l}32,58 \\
67,42\end{array}$ \\
\hline $\begin{array}{l}\text { Procedência } \\
\text { Pelotas } \\
\text { Capão do Leão } \\
\text { Outras** }\end{array}$ & $\begin{array}{c}200 \\
11 \\
10\end{array}$ & $\begin{array}{c}90,50 \\
4,98 \\
4,52\end{array}$ \\
\hline $\begin{array}{l}\text { Estado Civil } \\
\text { Com companheiro } \\
\text { Sem companheiro }\end{array}$ & $\begin{array}{l}141 \\
80\end{array}$ & $\begin{array}{l}63,80 \\
36,20\end{array}$ \\
\hline $\begin{array}{l}\text { Cor } \\
\text { Branca } \\
\text { Não branca }\end{array}$ & $\begin{array}{c}180 \\
41\end{array}$ & $\begin{array}{l}81,45 \\
18,55\end{array}$ \\
\hline $\begin{array}{l}\text { Escolaridade } \\
\text { Fundamental/médio incompleto } \\
\text { Médio completo/superior }\end{array}$ & $\begin{array}{l}175 \\
46\end{array}$ & $\begin{array}{l}79,19 \\
20,81\end{array}$ \\
\hline Comorbidades associadas ao DM2 & & \\
\hline $\begin{array}{l}\text { Hipertensão Arterial } \\
\text { Sim }\end{array}$ & 183 & 82,81 \\
\hline $\begin{array}{l}\text { Dislipidemia } \\
\text { Sim }\end{array}$ & 57 & 25,79 \\
\hline $\begin{array}{l}\text { Doença cardiovascular } \\
\text { Sim }\end{array}$ & 59 & 26,70 \\
\hline $\begin{array}{l}\text { Doença renal crônica } \\
\text { Sim }\end{array}$ & 33 & 14,93 \\
\hline
\end{tabular}

* Stata ${ }^{\circledR}$ versão 12.0. **Arroio Grande, Canguçu, Capão do Leão, Jaguarão, Pedro Osório e Tapes.

Em relação ao estado nutricional (Tabela 2), 59,55\% apresentaram obesidade, sendo as mulheres as que apresentaram maior percentual, com IMC médio de 32,83 $\pm 6,26$ $\mathrm{kg} / \mathrm{m}^{2}$. O peso médio entre os homens foi de $90,60 \pm 18,97 \mathrm{~kg}$ e entre as mulheres foi de $82,03 \pm 16,77 \mathrm{~kg}$, com diferença estatística significativa entre os sexos $(\mathrm{p}<0,05)$. 
Tabela 2 - Características antropométricas e estado nutricional, segundo o sexo, dos pacientes atendidos no Centro de Diabetes e Hipertensão da UFPel em Pelotas-RS, 2019 $(n=227)$

\begin{tabular}{|c|c|c|c|}
\hline & \multicolumn{3}{|c|}{ Sexo } \\
\hline Características * & $\begin{array}{c}\text { Masculino } \\
(n=72)\end{array}$ & $\begin{array}{c}\text { Feminino } \\
(n=149)\end{array}$ & Valor $p$ \\
\hline Peso (Kg) média $\pm d p^{* *}$ & $90,60 \pm 18,97$ & $82,03 \pm 16,77$ & 0,0008 \\
\hline IMC*** $\left(\mathrm{Kg} / \mathrm{m}^{2}\right)$ média $\pm d p$ & $31,71 \pm 5,85$ & $33,36 \pm 6,40$ & 0,0666 \\
\hline Classificação IMC n (\%) & & & \\
\hline Baixo peso & $1(25,00)$ & $3(75,00)$ & 0,257 \\
\hline Eutrofia & $14(46,66)$ & $16(53,34)$ & \\
\hline Sobrepeso & $19(34,55)$ & $36(65,46)$ & \\
\hline Obesidade & $38(28,24)$ & $94(71,76)$ & \\
\hline
\end{tabular}

*Teste t de student.**Dp= Desvio Padrão, ${ }^{* * *} \mathrm{IMC}=$ Índice de Massa Corporal.

Quanto à qualidade da dieta, a média de pontos do IQD-R foi de $60,63 \pm 9,88$, sendo $60,72 \pm 8,91$ para os homens e $60,43 \pm 10,34$ para mulheres. Os componentes do IQD$\mathrm{R}$ com os escores mais baixos foram "frutas integrais", "vegetais totais e leguminosas", "cereais integrais", "sódio", "frutas totais" e "leite e derivados", respectivamente. Já os maiores escores foram encontrados nos componentes que contemplam "gordura sólida, álcool e açúcar de adição", "óleos", "carnes, ovos e leguminosas", "gorduras saturadas", "cereais, raízes e tubérculos" e "vegetais verdes escuros, alaranjados e leguminosas", respectivamente (Tabela 3).

Tabela 3 - Escore médio de cada componente do IQD-R de pacientes diabéticos da cidade de Pelotas-RS, 2019 ( $n=221)$

\begin{tabular}{|c|c|c|c|}
\hline Componentes IQD-R & $\begin{array}{l}\text { Pontuação } \\
\text { Máxima }\end{array}$ & $\begin{array}{l}\text { Média } \\
\text { (pontos) }\end{array}$ & $\begin{array}{l}\text { Desvio } \\
\text { Padrão }\end{array}$ \\
\hline Frutas totais & 5 & 2,34 & 2,12 \\
\hline Frutas integrais & 5 & 0,63 & 1,59 \\
\hline $\begin{array}{l}\text { Vegetais totais e } \\
\text { leguminosas }\end{array}$ & 5 & 1,41 & 2,14 \\
\hline $\begin{array}{l}\text { Vegetais verdes escuros, } \\
\text { alaranjados e leguminosas }\end{array}$ & 5 & 3,13 & 2,40 \\
\hline $\begin{array}{l}\text { Cereais, raízes e } \\
\text { tubérculos }\end{array}$ & 5 & 3,95 & 1,42 \\
\hline Cereais integrais & 5 & 1,62 & 2,18 \\
\hline Leite e derivados & 10 & 3,49 & 3,58 \\
\hline $\begin{array}{l}\text { Carnes, ovos e } \\
\text { leguminosas }\end{array}$ & 10 & 8,89 & 2,27 \\
\hline Gordura Saturada & 10 & 6,51 & 3,52 \\
\hline Sódio & 10 & 1,97 & 2,58 \\
\hline Gord_AA* & 20 & 16,69 & 4,41 \\
\hline IQD-R** Total & 100 & 60,63 & 9,88 \\
\hline
\end{tabular}

*Gord_AA: calorias provenientes de gordura sólida (saturada e trans, álcool e açúcar de adição), **índice de Qualidade da Dieta Revisado: Para o componentes "óleos" foi considerado 10 pontos para todos os pacientes, em virtude do programa utilizado para avaliar o recordatório de 24 horas considerar um valor fixo para o óleo. 
De acordo com a Tabela 4, os indivíduos idosos apresentaram escores significativamente maiores de IQD-R quando comparados aos adultos (61,92 vs. 59,30 , respectivamente, $\mathrm{p}=0,0489$ ). Não houve, contudo, diferença estatística entre os escores do índice e as demais variáveis estudadas.

Tabela 4 - Associação do IQD-R e características dos pacientes atendidos no Centro de Diabetes e Hipertensão da UFPel em Pelotas-RS, 2019 ( $\mathrm{n=221)}$

\begin{tabular}{|c|c|c|}
\hline Características & $\begin{array}{c}\text { IQD-R* } \\
\text { (média } \pm d p)\end{array}$ & Valor $p$ \\
\hline $\begin{array}{l}\text { Sexo** } \\
\text { Masculino } \\
\text { Feminino }\end{array}$ & $\begin{array}{c}60,72 \pm 8,91 \\
60,43 \pm 10,34\end{array}$ & 0,8376 \\
\hline $\begin{array}{l}\text { Estado Civil** } \\
\text { Com companheiro } \\
\text { Sem companheiro }\end{array}$ & $\begin{array}{c}60,69 \pm 10,17 \\
60,23 \pm 9,41\end{array}$ & 0,7420 \\
\hline $\begin{array}{l}\text { Faixa etária** } \\
\text { Adulto } \\
\text { Idoso }\end{array}$ & $\begin{array}{c}59,30 \pm 10,04 \\
61,92 \pm 9,54\end{array}$ & 0,0489 \\
\hline $\begin{array}{l}\text { Escolaridade** } \\
\text { Fundamental/médio } \\
\text { incompleto } \\
\text { Médio completo/superior }\end{array}$ & $\begin{array}{l}60,29 \pm 9,74 \\
61,43 \pm 10,45\end{array}$ & 0,4850 \\
\hline $\begin{array}{l}\text { Estado Nutricional** } \\
\text { Baixo peso } \\
\text { Eutrofia } \\
\text { Sobrepeso } \\
\text { Obesidade }\end{array}$ & $\begin{array}{c}62,30 \pm 3,28 \\
63,04 \pm 9,05 \\
61,02 \pm 9,15 \\
59,76 \pm 10,43\end{array}$ & 0,3947 \\
\hline $\begin{array}{l}\text { Comorbidades** } \\
\text { Hipertensão Arterial } \\
\text { Sim } \\
\text { Não }\end{array}$ & $\begin{array}{c}60,64 \pm 10,20 \\
59,97 \pm 8,28\end{array}$ & 0,7063 \\
\hline $\begin{array}{l}\text { Dislipidemia } \\
\text { Sim } \\
\text { Não }\end{array}$ & $\begin{array}{c}60,04 \pm 10,37 \\
60,69 \pm 9,73\end{array}$ & 0,6709 \\
\hline $\begin{array}{l}\text { Doença Cardiovascular } \\
\text { Sim } \\
\text { Não }\end{array}$ & $\begin{array}{l}60,19 \pm 10,63 \\
60,65 \pm 9,62\end{array}$ & 0,7631 \\
\hline $\begin{array}{l}\text { Doença renal crônica } \\
\text { Sim } \\
\text { Não }\end{array}$ & $\begin{array}{l}62,59 \pm 8,30 \\
60,16 \pm 10,11\end{array}$ & 0,1938 \\
\hline
\end{tabular}

*índice de Qualidade da Dieta Revisado. ${ }^{* *}$ Teste t de student.

Não houve associação significativa entre melhor qualidade da dieta e estado nutricional, no entanto quando avaliado a correlação entre IMC e o IQD-R por meio da correlação de Pearson, os resultados demonstram uma correlação negativa significativa $(r=-0,16 ; p=0,0136)$, evidenciando que, quanto maior o IMC, menor o IQD-R. 


\section{DISCUSSÃO}

A qualidade da dieta em diabéticos é um dos fatores primordiais para a eficácia do tratamento e para uma boa qualidade de vida do paciente ${ }^{22}$. A partir disso esta pesquisa teve como objetivo aplicar o Índice de Qualidade da Dieta Revisado em uma amostra de indivíduos com DM2 atendidos em um centro de referência do Sul do Brasil.

Foi apurado que a média geral de idade da amostra estudada foi de 58 anos, sendo constatada a presença significativamente maior de mulheres. Este dado foi encontrado em outros estudos, nos quais ficou evidenciado que o sexo feminino tende a ter uma busca constante por assistência à saúde e uma preocupação superior com a qualidade da dieta, aspectos associados à inserção cada vez maior das mulheres no mercado de trabalho gerando cada vez mais autonomia para a aquisição de insumos alimentícios de qualidade em virtude da saúde ${ }^{23-27}$. Observou-se ainda que a maioria dos pacientes era da cor branca, possuía companheiro e apresentava ensino fundamental completo ou médio incompleto além de diagnóstico de hipertensão arterial. O último dado também foi encontrado em vários estudos ${ }^{28-30}$, comprovando a prevalência elevada das duas doenças isoladas, além da associação entre elas.

Em relação ao estado nutricional, foi observado que ambos os sexos apresentaram prevalência de obesidade, porém as mulheres apresentaram maiores percentuais de IMC e os homens obtiveram maior peso sendo evidenciada a diferença de peso estatisticamente entre os sexos. Zanchim et al. ${ }^{31}$ avaliaram os marcadores do consumo alimentar em 100 pacientes diabéticos internados em um hospital do interior do estado do Rio Grande do Sul, e também observaram que os pacientes apresentaram na sua maioria sobrepeso e obesidade vinculada ao diabetes, sendo um importante fator que predispõe a altos percentuais de morbidade e mortalidade. Resultados semelhantes foram encontrados por Rossaneis et al. ${ }^{32}$, que investigaram diferenças no autocuidado com o estilo de vida em 1.515 diabéticos de ambos os sexos. Segundo os autores, as mulheres apresentaram média de IMC maior do que os homens, sendo classificadas com excesso de peso. Outro estudo associou este fato com hábitos alimentares inadequados podendo ser responsáveis pela alta prevalência de obesidade, especificamente no sexo feminino ${ }^{33}$.

Em relação à qualidade da dieta, a média de pontos do IQD-R foi de $60,63 \pm 9,88$ pontos. Resultado semelhante foi verificado por Fisberg et al. ${ }^{34}$, por meio de um 
estudo transversal, avaliando a qualidade da dieta e os fatores associados em 3.454 adultos com 20 anos ou mais, residentes do estado de São Paulo, em que foram identificados 60,4 pontos como média do escore do índice de qualidade da dieta (IQD) - versão anterior ao IQD-R. Similarmente Assumpção et al. ${ }^{35}$ em um estudo transversal, em que foi avaliada a qualidade da dieta por meio do IQD-R em uma amostra de 1.509 idosos, obtiveram como resultado o valor médio de 62,4 pontos. Campos et al. ${ }^{36}$ avaliaram a qualidade da dieta de docentes de uma instituição de ensino superior de Belém, sendo avaliados 23 indivíduos e verificou-se que a média de pontos do IQD-R foi de 66,43 pontos. A classificação do presente índice ${ }^{18}$ classifica a dieta com pontuação mais próxima dos 100 pontos com uma qualidade superior. Com o intuito de classificar essa qualidade da dieta com mais clareza foi utilizado a classificação do IQD anterior ${ }^{17}$, que classifica a dieta em três categorias: abaixo ou igual a 40 pontos - dieta "inadequada"; entre 41 e 64 pontos - dieta que "necessita de modificação"; e igual ou superior a 65 pontos - dieta "saudável". Podemos afirmar que os pacientes diabéticos apresentam uma dieta que "necessita de modificação", salientando a importância do acompanhamento nutricional.

Os componentes com menor contribuição na média para o escore total do IQD-R foram "frutas integrais", "vegetais totais e leguminosas", "cereais integrais, "frutas totais", "leite e derivados" e sódio. Assumpção et al. ${ }^{37}$, ao avaliarem o consumo alimentar por meio do IQD-R em homens e mulheres, obtiveram menor pontuação nos mesmos componentes. Na literatura brasileira ainda foi encontrado outro estudo ${ }^{38}$ que teve como objetivo caracterizar a qualidade e os fatores associados à dieta de mulheres com excesso de peso, sendo observada menor adequação nos itens sódio, cereais integrais e leite e derivados, em encontro do presente estudo.

Freedman et al. ${ }^{39}$ observaram que, na população americana, os componentes de frutas totais e frutas inteiras estavam abaixo dos valores máximos de pontuação para estes componentes. Ervin et al. ${ }^{\circ 0}$ em seu estudo norte-americano sobre consumo alimentar, encontraram baixo consumo de vegetais totais, sendo consonante com os resultados de Nelson et al. ${ }^{41}$, que descreveram as práticas de dieta alimentar de indivíduos com DM2 autorreferida. Segundo a Pesquisa Nacional de Saúde e Nutrição com indivíduos diabéticos, foi encontrada baixa adesão às recomendações de frutas e vegetais, isto é, $62 \%$ dos entrevistados comiam menos de cinco porções de frutas e vegetais por dia. Indo em consonância com a Pesquisa de Orçamento Familiar (POF) de 2008/200942, observou-se que o brasileiro consome 
frutas e vegetais em uma quantidade muito abaixo das suas necessidades. Muitos pacientes diabéticos possuem uma interpretação errônea em relação ao açúcar que contém nas frutas, logo evitando consumi-las. Entretanto o açúcar presente nas frutas (frutose) é diferente do adicionado a doces e refrigerantes, além da fruta conter fibras na sua composição. Assim o processo de absorção no organismo ocorre lentamente, evitando picos glicêmicos ${ }^{43}$.

Em relação ao componente "cereais integrais", vários estudos obtiveram como resultado a ingestão inadequada, desse item $37,38,44$. Kuznesof et al. ${ }^{45}$ descrevem que as principais barreiras para a ingestão adequada de cereais integrais seriam a baixa aceitação dos alimentos pela família, habilidades culinárias, condição financeira e baixa disponibilidade de produtos integrais nos mercados. Alimentos integrais têm um papel de extrema relevância no manejo dietoterápico para pacientes com diabetes, tendo efeitos benéficos sobre a homeostase glicêmica, além de ter efeitos positivos sobre saciedade, peso corporal, melhora do sistema imunológico e redução dos fatores de risco para doenças cardiovasculares $44,46,47$.

No presente estudo a média de pontos para "leite e derivados" foi de 3,49 pontos evidenciando a inadequação no consumo deste componente. De acordo com Previdelli et al. ${ }^{18}$, o consumo adequado seria 1,5 porção/100o kcal (10 pontos). Assumpção et al. ${ }^{35}$ encontraram uma média de 4,90 pontos para o componente leite e derivados, indo em concordância com outro estudo realizado pelo mesmo pesquisador ${ }^{37}$, onde demonstrou que no geral a média nesse componente foi de 4,12 pontos, evidenciando a baixa adesão a este componente. O consumo de leite e derivados é essencial para suprir a recomendação de cálcio e prevenção da osteoporose, no entanto, observa-se que apesar da recomendação preconizada pelo Guia Alimentar para a População Brasileira ${ }^{21}$ ser o consumo de três porções de leite e produtos lácteos diariamente, no Brasil, há uma tendência de redução do seu consumo 35 .

O componente sódio contribuiu com uma pontuação baixa (média de 1,97 pontos) para o escore final do IQD-R. Em concordância, Assumpção et al.35,37 obtiveram em seus dois estudos uma baixa pontuação para este componente (média de 2,10 pontos e média de 2,27 pontos, respectivamente), assim com Loureiro et al..$^{8}$ (média de 2,43 pontos). Este componente avalia a moderação no consumo, ou seja, a maior parte dos pacientes extrapolou o consumo de sódio ( $\geq 2,0 \mathrm{~g} / 1.000 \mathrm{kcal})$, resultando em uma baixa pontuação. $O$ adequado consumo de sódio segundo o estudo base sobre o IQD- 
$\mathrm{R}$ para a população brasileira ${ }^{18}$, seria $0,75 \mathrm{~g} / 1.000 \mathrm{kcal}$. Salientando que o consumo de sódio, além do recomendado, é um fator de risco importante para a ocorrência de hipertensão arterial e doenças cardiovasculares ${ }^{28}$.

Já os componentes com maiores escores foram "gordura sólida, álcool e açúcar de adição", "óleos", "carnes, ovos e leguminosas", "gorduras saturadas", "cereais, raízes e tubérculos" e "vegetais verdes escuros, alaranjados e leguminosas", respectivamente. O componente "gordura sólida, álcool e açúcar de adição", que engloba alimentos considerados fontes de "calorias vazias", ou seja, apresenta um elevado conteúdo energético e uma baixa oferta de nutrientes, obteve nesta amostra a média de 16,69 pontos. Loureiro et al. ${ }^{48}$ encontraram uma média de pontos semelhante (16,16 pontos), sendo superior aos achados de Assumpção et al. ${ }^{35}$ que encontraram uma média de 12,5 pontos. Em um estudo realizado por Oliveira et al. ${ }^{49}$, que avaliou o efeito de orientação individual nas mudanças das práticas alimentares, verificou-se uma redução do consumo de gordura sólida, álcool e açúcar de adição após os pacientes receberem orientações.

Pressupõe-se que os indivíduos do presente estudo podem ter tido algum tipo de orientação anteriormente, assim consumindo uma menor quantidade dos alimentos que integram esse componente. Sabe-se que o consumo exacerbado destes alimentos, acarreta uma série de alterações no metabolismo, levando ao acúmulo de gordura visceral, que elevam o risco hipertensão arterial e das taxas de colesterol no sangue, podendo ocasionar outras doenças em conjunto com o DM2, levando a complicações graves ${ }^{1,3}$.

Para o componente "óleos" foi considerada uma pontuação máxima para todos os pacientes, isto é, 10 pontos, em decorrência do programa utilizado para avaliar o recordatório de 24 horas considerar um valor fixo para esse componente, levando em conta toda a quantidade de ácidos graxos consumida (monoinsaturadas, poliinsaturadas, Ômega 3 e Ômega 6). A partir disso o valor fixo definido foi de 16 $\mathrm{ml}$, equivalente a duas porções, deste modo excedendo a porção máxima indicada por Previdelli et $\mathrm{al}^{18}$ (0,5 porção/1.00o kcal). Em concordância alguns estudos também demonstraram escore máximo para o componente "óleos"38,50.

Entre os nove componentes dos grupos alimentares, as "carnes, ovos e leguminosas" e os "cereais, raízes e tubérculos" obtiveram médias superiores comparadas aos outros componentes: 8,89 pontos e 3,9 pontos respectivamente. Campos et al. ${ }^{36}$ encontraram resultados similares, e os grupos mais consumidos 
foram "Cereais Totais" (com média de 4,35 pontos) e "Carnes, ovos e Leguminosas" (com média de 8,73 pontos), podendo ser encontrados mais resultados semelhantes em outros estudos que utilizaram o instrumento IQD-R $\mathrm{R}^{35,51}$. Benetti et al.52, que investigaram a frequência do consumo alimentar de pacientes diabéticos em um ambulatório de especialidades em nutrição, também observaram uma maior ingestão de alimentos do grupo dos cereais, assim como uma ingestão semanal variada de carnes e um consumo significativo de feijão.

É importante ressaltar que o índice utilizado no presente estudo englobou as leguminosas no grupo das carnes, assim contribuindo para um alto escore desse componente. Devido ao hábito alimentar brasileiro de consumir feijão, sua inclusão neste grupo pode levar a superestimação da ingestão33. Os grupos das "carnes, ovos e leguminosas" e dos "cereais, raízes e tubérculos", devem ter seu consumo moderado para pacientes DM2 dentro de uma dieta saudável, pois é importante lembrar que o primeiro componente citado envolve proteínas, e aproximadamente $35 \%$ a $60 \%$ do total de proteína ingerida converter-se em glicose, e consequentemente pode elevar a glicemia, caso seu consumo seja excessivo. $\mathrm{O}$ segundo componente citado envolve carboidratos, e os alimentos pertencentes à este grupo contém uma quantidade significativa de carboidrato podendo ocasionar picos glicêmicos, que pode levar a consequências negativas se persistirem por longo período ${ }^{6,7,47}$.

Já o grupo dos "vegetais verdes escuros, alaranjados e leguminosas" obteve uma pontuação de 3,13 pontos, em concordância com o achado no estudo de Assumpção et al. ${ }^{35}$, que encontraram um consumo médio de 3,9 pontos. Este grupo por serem ricos em vitaminas, minerais e compostos antioxidantes, atuam como protetores de futuras complicações do diabetes, tendo efeitos positivos, principalmente na redução do estresse oxidativo ${ }^{3,8}$.

Em relação à gordura saturada, a pontuação obtida foi de 6,51 pontos, atingindo em média $8,10 \%$ do Valor Energético Total (VET). Segundo Previdelli et al. ${ }^{18}$ o consumo ideal de gorduras saturadas seria $<7 \%$ do VET, tendo esse embasamento nas diretrizes sobre dislipidemias e prevenção da aterosclerose da Sociedade Brasileira de Cardiologia ${ }^{53}$. O consumo elevado deste componente para o paciente diabético pode causar elevação dos níveis de colesterol e triglicerídeos. Campos et al. ${ }^{36} \mathrm{e}$ Assumpção et al. ${ }^{37}$ obtiveram pontuações maiores nesse componente, 8,73 pontos e 8,37 pontos respectivamente. 
Observou-se que os indivíduos idosos apresentaram escores maiores de IQD-R quando comparados aos adultos. Hiza et al..$^{51}$ avaliaram a qualidade da dieta de americanos, utilizando o Healthy Eating Index- 2005, e encontraram que os idosos tinham dietas de melhor qualidade, corroborando com os achados de Assumpção et al. ${ }^{35}$. Possivelmente a melhor qualidade da dieta de idosos esteja associada à presença de doenças crônicas, além de uma mudança de consciência sobre saúde com o aumento da idade, o que leva à adoção de hábitos mais saudáveis ${ }^{54}$. Outra justificativa seria que os padrões alimentares de antigamente eram diferentes dos observados atualmente. Os idosos dos dias atuais foram menos expostos a alimentos processados e ultraprocessados, quando formaram seus hábitos alimentares, deste modo influenciando nas suas escolhas alimentares ${ }^{55}$.

Não houve diferença significativa entre a qualidade da dieta e as variáveis sexo, estado civil, escolaridade e comorbidades. Em oposição, outros estudos verificaram melhor qualidade da dieta em mulheres, indivíduos casados, com maior escolaridade e uma associação positiva com doenças crônicas, ou seja, quanto mais doenças crônicas associadas o indivíduo tiver, maior qualidade da dieta35,37,49,56-58.

No que se refere ao estado nutricional, não houve associação entre melhor qualidade da dieta e estado nutricional, porém quando avaliada a correlação entre eles por meio da correlação de Pearson, evidenciou-se que quanto maior o IMC, menor o IQD-R. Freitas et $a .^{58}$ realizaram um estudo transversal onde avaliaram os fatores associados à qualidade da dieta de 295 idosos da cidade de São Caetano do Sul, Brasil. Segundo os autores foi encontrado associação entre o estado nutricional e o escore do IQD-R, mostrando que idosos obesos apresentaram menores escores de IQD-R. Em um estudo ${ }^{59}$ de coorte norte-americano observou-se que a maior qualidade da dieta estaria associada a um menor IMC. Coltman et al..$^{60}$ que comparou o consumo alimentar de não obesos e obesos, observou-se que os indivíduos não obesos obtiveram escores significativamente mais altos em vegetais verdes-escuros, alaranjados e leguminosas, cereais totais e cereais integrais. É possível concluir que, quando os indivíduos tem uma preferência pelos alimentos que compõem os grupos citados acima, existe uma maior probabilidade de maior qualidade da dieta, assim contribuindo para um peso adequado.

O instrumento utilizado nesse estudo permite um amplo conhecimento da dieta da população estudada, uma vez que avalia a qualidade da dieta, independente da quantidade de alimentos consumida ${ }^{18}$. Por se tratar de um índice recentemente 
adaptado para a população brasileira, são escassos os estudos que avaliem o IQD-R especificamente em diabéticos, evidenciando este estudo como um dos pioneiros abordando esta temática.

A principal limitação deste trabalho refere-se ao instrumento utilizado para a avaliação do consumo alimentar, ou seja, o recordatório de 24 horas, aplicado em um único dia, podendo não refletir o consumo habitual. $\mathrm{O}$ ideal seria aplicar três recordatórios de 24 horas. Outra limitação refere-se ao tamanho da amostra, que pode ter prejudicado o encontro de associações significativas entre as variáveis estudadas por falta de poder estatístico.

\section{CONCLUSÃO}

Mediante o presente estudo, verificou-se que a população estudada apresentou uma necessidade de modificação de sua dieta, evidenciando uma inadequação do consumo alimentar, principalmente pela menor pontuação nos componentes que avaliaram o consumo de frutas, vegetais, cereais integrais, sódio e leite e derivados. Desta forma subentende-se que não foram atendidas as recomendações presentes no Guia Alimentar Brasileiro para os componentes citados anteriormente.

Conclui-se que há necessidade de intervenções nutricionais no intuito de promover uma melhor qualidade da dieta deste público. Ressalta-se ainda, que o consumo alimentar adequado para pacientes diabéticos é um dos principais pilares para garantir qualidade de vida, melhora nos hábitos alimentares e redução de complicações associadas a esta doença.

\section{REFERÊNCIAS}

1. World Health Organization. The top 10 causes of death [Internet]. Geneva: World Health Organization; 2018. Acesso: 26 mar. 2020. Disponível em:

http://www.who.int/news-room/fact-sheets/detail/the-top-10-causes-ofdeath.

2. International Diabetes Federation. IDF Diabetes Atlas. 9 th ed. [Internet]. Brussels: International Diabetes Federation; 2019. Acesso em: 26 mar. 2020. Disponível em: https://www.diabetesatlas.org/en/sections/demographic-andgeographic-outline.html.

3. International Diabetes Federation. IDF Diabetes Atlas. 8 th ed. [Internet]. Brussels: International Diabetes Federation; 2017. Acesso em: 26 set. 2020. Disponível em: http://www.diabetesatlas.org/across-the-globe.html.

4. Costa A, Flor L, Campos M, Oliveira A, Costa Maria, Silva R et al. Carga do diabetes mellitus tipo 2 no Brasil. Cad. Saúde Pública [Internet]. 2017; 33 (2): e00197915. 
5. Assunção SC, Fonseca AP, Silveira MF, Caldeira AP, Pinho L. Knowledge and attitude of patients with diabetes mellitus in Primary Health Care. Esc. Anna Nery. Rio de Janeiro; 2017; 21(4): 170-208.

6. Brasil. Ministério da Saúde. Secretaria de Atenção à saúde. Departamento de Atenção Básica. Diabetes Mellitus: Caderno de Atenção Básica, n 16 Série A [Internet]. Brasília- DF: Normas e Manuais Técnicos, 2006. Acesso: 19 set. 2018. Disponível em:

http://bvsms.saude.gov.br/bvs/publicacoes/diabetes_mellitus_cab16.pdf.

7. Sociedade Brasileira de Diabetes. Diretrizes da Sociedade Brasileira de Diabetes (SBD), Diretrizes da Sociedade Brasileira de Diabetes (2017-2018). São Paulo; 2017. Acesso: 19 set. 2018. Disponível em:

https://www.diabetes.org.br/profissionais/imagens/2017/diretrizes/diretrizessbd 2017-2018.pdf.

8. Ramírez MH, Flores CAJ, Alvarado MRB, Cuahutle ADO, Vicente IAH, Tarabay JAB. Promotion of a healthy lifestyle in individuals with type 2 diabetes mellitus in rural communities. Rev. Horiz. Sanitario. Villahermosa; 2017; 16(3): 191-200.

9. Guerreiro-Pacheco R, Galan-Cuevas S, Capello OSA. Sociodemographic and psychological factors associated with self-care and quality of life in Mexican adults with type 2 Diabetes Mellitus. Acta. colomb. psicol. Bogotá; 2017; 20(2): 168-177.

10. Sartorelli DS, Franco, LJ. Tendências do diabetes mellitus no Brasil: o papel da transição nutricional. Cad. Saúde Pública. Rio de Janeiro; 2003; 19(1): 29-36.

11. Gross LS, Li L, Ford ES, Liu S. Increased consumption of refined carbohydrates and the epidemic of type 2 diabetes in the United States: an ecologic assessment. Am J Clin. Nutr. 2004; 79(5): 774-779.

12. Santos CRB, Gouveia LAV, Portella ES, Ávila SS, Soares EA, Lanzillotti HS. Índice de Alimentação Saudável: avaliação do consumo alimentar de diabéticos tipo 2. Rev. Soc. Bras. Alim. Nutr. São Paulo; 2009; 34(1): 115-129.

13. Felippe F, Balestrin L, Silva FM, Schneider AP. Qualidade da dieta de indivíduos expostos e não expostos a um programa de reeducação alimentar. Rev. Nutr. Campinas; 2011; 24(6): 833-844.

14. Kennedy ET, Ohls J, Carlson S, Fleming K. The Healthy Eating Index: design and applications. J Am Diet Assoc. 1995; 95(10): 1103-1108.

15. Guenther PM, Casavale KO, Kirkpatrick SI, Reedy J, Hiza HAB, Kucznski KJ et al. Update of the Healthy Eating Index: HEI-2010. J Acad. Nutr. Diet. 2013; 113(4): 569-580.

16. Fisberg RM, Slater B, Barros RR, Lima FD, Cesar CFG, Carandina L et al. Índice de Qualidade da Dieta: avaliação da adaptação e aplicabilidade. Rev. Nutr. Campinas; 2004; 17(3): 301-318.

17. Philippi ST, Latterza AR, Cruz ATR, Ribeiro LC. Pirâmide alimentar adaptada: guia para escolha dos alimentos. Rev. Nutr. Campinas; 1999; 12(1): 65-80.

18. Previdelli AN, Andrade SC, Pires MM, Ferreira SRG, Fisberg RM, Marchioni DM. Índice de Qualidade da Dieta Revisado para população brasileira. Rev. Saúde Pública. São Paulo; 2011; 45(4): 794-798. 
19. World Health Organization. The world health report 1997[Internet]. Conquering suffering, enriching humanity. Geneva: World Health Organization. Acesso: 19 out. 2018. Disponível em: https://www.who.int/whr/1997/en/.

20. Sass A, Marcon SS. Comparação de medidas antropométricas de idosos residentes em área urbana no sul do Brasil, segundo sexo e faixa etária. Rev. Bras. Geriatr. Gerontol. Rio de Janeiro; 2015; 18(2): 361-372.

21. Brasil. Ministério da Saúde. Secretaria de Atenção à Saúde. Departamento de Atenção Básica. Guia alimentar para a população brasileira / Ministério da Saúde, Secretaria de Atenção à Saúde, Departamento de Atenção Básica. Brasília; 2014; 2(1). Acesso: 20 nov. 2018. Disponível em:

http://bvsms.saude.gov.br/bvs/publicacoes/guia_alimentar_populacao_brasile ia_2ed.pdf

22. Fernandes CAM, Nardo NJ, Tasca RS, Pelloso SM, Cuman RKN. A importância da associação de dieta e de atividade física na prevenção e controle do Diabetes mellitus tipo 2. Acta Sci. Health Sci. 2005; 27(2): 195-205.

23. Oliveira KCS, Zanetti ML. Conhecimento e atitude de usuários com diabetes mellitus em um serviço de Atenção Básica à Saúde. Rev. Esc. Enferm. USP. São Paulo, 2011; 45(4): 862-868.

24. Levorato CD, Melo LM, Soares AS, Nunes AA. Fatores associados à procura por serviços de saúde numa perspectiva relacional de gênero. Ciênc. Saúde Coletiva. Rio de Janeiro 2014.19(4).

25. Cruz ARR, Melo CMA, Barbosa CO. Conhecimentos dos diabéticos sobre a doença e o tratamento nutricional. RBCEH. 2011; 8(3): 343-354.

26. Oliveira TRPR, Pereira GP. Perfil de pacientes que procuram a Clínica de Nutrição da PUC MINAS e satisfação quanto ao atendimento: percurso acadêmico. Rev. Interdiscip. PUC. Minas no Barreiro 2014; 4(8): 268-282.

27. Grillo MFF, Gorini MIPC. Caracterização de pessoas com diabetes mellitus Tipo 2. Rev. Bras. Enferm. 2007; 1(60): 49-54.

28. Silva DB, Souza TA, Santos CM, Jucá MM, Moreira TMM, Frota MA et al. Associação entre Hipertensão Arterial e Diabetes em Centro de Saúde da Família. RBPS. Fortaleza, 2011; 24(1): 16-23.

29. Schmidt MI, Duncan BB, Hoffman JF, Moura L, Malta DC, Carvalho RMS. Prevalência de diabetes e hipertensão no Brasil baseada em inquérito de morbidade auto-referida, Brasil. Rev. Saúde Pública. 2009; 43(2): 74-82.

30. Souza MFC, Araújo VF. Adequação do consumo e evolução antropométrica após educação nutricional de pacientes com diabete mellitus tipo 2. Rev. Demetra. 2015; 13(1): 159-172.

31. Zanchin MC, Kirsten VR, Marchi ACB. Marcadores do consumo alimentar de pacientes diabéticos avaliados por meio de um aplicativo móvel. Ciênc. Saúde Coletiva. 2018; 23(12): 4199-4208.

32. Rossaneis MA, Haddad MCFL, Mathias TAF, Marcon SS. Diferenças entre mulheres e homens diabéticos no autocuidado com os pés e estilo de vida. RLAE. 2016; 24:e2761.

33. Costa D, Reis BZ, Vieira DAS, Costa JO, Teixeira PDS, Raposo OFF et al. Índice de qualidade da dieta de mulheres usuárias de um programa de atividade física 
regular "Academia da Cidade", Aracajú, SE. Rev. Nutr. Campinas, 2012; 25(6): 731-741.

34. Fisberg RM, Morimoto JM, Slater B, Barros MB, Carandina L, Goldbaum M. Dietary quality and associated factors among adults living in the state of. J. Acad. Nutr. Diet. São Paulo, 2006; 106(12): 2067-72.

35. Assumpção D, Domene SMA, Fisberg RM, Barros MBA. Qualidade da dieta e fatores associados entre idosos: estudo de base populacional em Campinas, São Paulo, Brasil. Cad. Saúde Pública. Rio de Janeiro, 2014, 30(8): 1680-1694.

36. Campos JSP, Borges SM. Avaliação da qualidade da dieta e fatores de risco à saúde de docente de uma instituição de ensino superior de Belém - PA. Braspen J. 2018; 33(2): 147-51.

37. Assumpção D, Domene SMA, Fisberg RM, Canesqui AM, Barros MBA. Diferenças entre homens e mulheres na qualidade da dieta: estudo de base populacional em Campinas, São Paulo. Ciênc. Saúde Coletiva. Rio de Janeiro, 2017, 22(2): 347-358.

38. Horta PM, Santos LC. Qualidade da dieta entre mulheres com excesso de peso atendidas em uma Academia da Cidade de Belo Horizonte, Minas Gerais. Cad. Saúde Colet. Rio de Janeiro, 2015, 23(2): 206-213.

39. Freedman LS, Guenther PM, Krebs-Smith SM, Kott PS. A population's mean Healthy Eating Index-2005 scores are best estimated by the score of the population ratio when one 24 -hour recall is available. J. Nutr. 2008; 138(9): 1725-1729.

40. Ervin RB. Healthy Eating Index-2005 total and component scores for adults aged 20 and over: National Health and Nutrition Examination Survey, 20032004. NHIS. 2011; 13(44): 1-9.

41. Nelson KM, Reiber G, Boyko EJ, Nhanes III. Diet and exercise among adults with type 2 diabetes: findings from the third national health and nutrition examination survey. Diabetes Care. 2002; 25: 1722-1728.

42. IBGE. Pesquisa de Orçamentos Familiares 2008-2009: despesas, rendimentos e condições de vida. Rio de Janeiro: IBGE, 2010. Acesso: 09 maio de 2019. Disponível em: https://www.ibge.gov.br/estatisticas/sociais/saude/9050pesquisa-de orcamentos-familiares.html?=\&t=resultados.

43. Silva ME, Simões MO, Almeida KSM. Qualidade da dieta e consumo de sódio de pacientes em hemodiálise de uma clínica renal da fronteira oeste do Rio Grande do Sul. Rev. Bras. Nutr. Clín. 2016; 31 (1): 70-74.

44. Bernaud FSR, Rodrigues TC. Fibra alimentar - Ingestão adequada e efeitos sobre a saúde do metabolismo. ABE\&M. 2013; 57(6): 397-405.

45. Kuznesof S, Brownlee IA, Moore C, Richardson DP, Jebb AS, Seal CJ. Whole heart study participant acceptance of wholegrain foods. Appetite. 2012; 59(1): 187-93.

46. Dall'Alba V, Azevedo MJ. Papel das fibras alimentares sobre o controle glicêmico, perfil lipídico e pressão arterial em pacientes com diabetes melito tipo 2 . Rev. HCPA. 2010; 30(4): 363-371.

47. Anderson JW, Randles KM, Kendall CW, Jenkins DJ. Carbohydrate and fiber recommendations for individuals with diabetes: a quantitative assessment and meta-analysis of the evidence. J. Am. College Nutr. 2004; 23(1): 5-17. 
48. Loureiro AS, Silva RMVG, Rodrigues PRM, Pereira RA, Wendpap LL, Ferreira MG. Diet quality in a sample of adults from Cuiabá (MT), Brazil: Association with sociodemographic factors. Rev. Nutr. Cuiabá, 2013, 26(4): 431-441.

49. Oliveira MC, Calahani IM, Previdelli AN, Malta MB, Corrente JE, Papini SJ. Aplicação do Índice de Qualidade da Dieta Revisado antes e após orientação nutricional. Rev. Ciênc. Méd. 2015, 24(1): 29-36.

50. Ceccatto V, Pietro PF, Previdelli AN, Vieira FGK, Schiavon CC, Engel R. Brazilian Healthy Eating Index Revised (BHEI-R) of women before and during adjuvant treatment for breast cancer. Nutr. Hosp. 2014; 30(5): 1101-1109.

51. Hiza HA, Casavale KO, Guenther PM, Davis CA. Diet quality of Americans differs by age, sex, race/ethnicity, income, and education level. J. Acad. Nutr. Diet. 2013; 113(2): 297-306.

52. Benetti F, Ceni GC. Frequência do consumo alimentar de pacientes diabéticos em acompanhamento ambulatorial na região norte do RS, no segundo semestre de 2008. Rev. Eletrôn. de Extensão da URI. Vivências, 2010; 6(9): 124-135.

53. Sociedade Brasileira de Cardiologia. IV Diretriz Brasileira sobre dislipidemias e prevenção da aterosclerose. Arquivo Brasileiro de Cardiologia. 2007; 88(1): 2-19.

54. Tardido AP, Falcão MC. O impacto da modernização na transição nutricional e obesidade. Rev. Bras. Nutr. Clín. 2006, 21(2): 117-124.

55. Pereira IFS, Vale D, Bezerra MS, Lima KC, Roncalli AG, Lyra CO. Padrões alimentares de idosos no Brasil: Pesquisa nacional de saúde. Ciên. Saúde Colet. 2013.

56. Assumpção D, Senicato C, Fisberg RM, Canesqui AM, Barros MBA. Há diferenças na qualidade da dieta de trabalhadoras remuneradas e donas de casa? Rev. Saúde Pública. São Paulo, 2018, 52(47).

57. Chen X, Cheskin LJ, SHI L, WANG Y. Americans with diet-related chronic diseases report higher diet quality than those without these diseases. J. Nutr. 2011, 141(8):1543-1551.

58. Freitas TI, Previdelli AN, Ferreira MPN, Marques KM, Goulart RMM, Aquino RC. Factors associated with diet quality of older adults. Rev. Nutr. Campinas, 2017, 30(3): 297-306.

59. De Koning L, Chiuve SE, Fung TT, Willett WC, Rimm EB, Hu FB. Diet-Quality Scores and the Rick of Type 2 Diabetes in Men. Diabetes care. 2011; 34(5): 11501156.

60. Coltman AE, Keim KS, Chapman-Novakofski KM, Taylor CA. Assessing Diet Quality of a Type 2 Diabetes Sample Using the Healthy Eating Index 2005. TICN. 2013; 28(2): 145-153.

Submissão: 04/08/2019

Aprovação: 23/07/2020 